\title{
Clinical and epidemiological study of scorpion sting envenomation at a teaching hospital in rural Telangana
}

\author{
Reddy R.M. ${ }^{1}$, G. Somaiah ${ }^{2}$ \\ ${ }^{1}$ Dr. Rukmajigari Manohar Reddy, Assistant Professor, Department of Pediatrics, Maheshwara Medical College \\ \& Hospital, Patancheru, Telangana, ${ }^{2}$ Dr. G. Somaiah, Professor, Department of Pediatrics, Mamata Medical \\ College \& Hospital, Khammam,Telangana.
}

Address for Correspondence: Dr. Rukmajigari Manohar Reddy, Assistant Professor, Department of Pediatrics, Maheshwara Medical College \& Hospital, Patancheru, Telangana. E-mail Id: docmic175@gmail.com, resdoc555@gmail.com

\begin{abstract}
Introduction: Scorpions have adapted to diverse environments and are mainly found in tropical and sub- tropical countries, between latitude $50 \mathrm{~N}$ and $50 \mathrm{~S}$. Scorpion stings are primarily due to accidental contact with scorpion. Scorpions capable of inflicting fatal stings in humans are all members of families Buthus and Scorpionidae. Reports from Bellary, Rayalaseema, rural Maharashtra, Pondicherry and Chennai have documented such fatalities in children and adults. This study was done to know the clinical presentation, course, complications and outcome of scorpion sting envenomation. To study the epidemiology and circumstances leading to scorpion sting in the community. Materials and Methods: 50 cases of scorpion sting, admitted to Mamata General Hospital, Khammam from October 2010 to October 2012 were included in the study. On admission, a detailed clinical history, including the time of sting, symptomatology, details of treatment received before admission was taken. Further a description of the scorpion and details about the circumstances leading up to the sting were obtained. Results: Maximum numbers of cases were noted in the age groups of 4-6 years. Scorpion sting were more common in males. Maximum number of cases were seen in rainy and in the summer season. Stings due to Mesobuthus species (Red scorpion) were slightly more common than stings due to Palamneus species (Black scorpion). Conclusion: Scorpions have been known to man since time immemorial and have a significant presence in mythology and human history. Scorpion stings, once considered just a painful nuisance have now got the attention and care that they deserve. Early and effective prazosin therapy, good supportive care, close monitoring and management of complications can limit the resulting morbidity and mortality significantly. The role of scorpion antivenom still remains controversial.
\end{abstract}

Key words: Scorpion, Sting, Envenomation, Complications

\section{Introduction}

Out of 1500 scorpion species, 50 are dangerous to humans. Scorpions are found on all major land masses except Antarctica. Scorpions do not occur naturally in Great Britain, New Zealand and some of the islands in Oceania, but have now been accidentally introduced in some of these places by human trade and commerce. Scorpions have adapted to diverse environments and are mainly found in tropical and sub tropical countries, between latitude $50 \mathrm{~N}$ and $50 \mathrm{~S}[1,2]$. South and Central America,

Manuscript received: $4^{\text {th }}$ July 2017

Reviewed: $14^{\text {th }}$ July 2017

Author Corrected: $22^{\text {nd }}$ July 2017

Accepted for Publication: $30^{\text {th }}$ July 2017
Southern United States, countries of the Arabian peninsula, Northern Africa and part of the Indian sub-continent are endemic for scorpions [1]. In Asia epidemiological data on scorpion stings is scarce. India is the most affected, with a reported incidence of $0.6 \%$. A retrospective analysis of the calls received by the National Poison Information Center (NPIC) between April 1999 and March 2002 showed that, out of 995 calls, 6 involved scorpion stings. During hot months March to June and September to October daily cases of severe scorpion sting were received at endemic areas western Maharashtra, Karnataka, Andhra Pradesh, 
Saurashtra and Tamil Nadu. Severe scorpion sting due to Mesobuthus tumulus species of scorpion per month reported from Konkan region. Higher incidence of scorpion sting occurred during hot months attributed to increase in agriculture activities [3].

Scorpions live in warm dry regions throughout India. They inhabit commonly the crevices of dwellings, underground burrows, under logs or debris, paddy husk, sugarcane fields, coconut and banana plantations. Their distribution is more in regions with abundant red soil. Scorpions may be found outside their natural range of distribution when inadvertently transported with other items such as luggage. The Scorpion is primarily a nocturnal arthropod. Scorpions retreat in the crevices of dwellings during the day only to emerge at night; thus most stings are reported at night. They hunt insects, crickets, small lizards and even other scorpions! Scorpions are active in summer and hibernate in winter, so scorpion stings increase dramatically in summer months and are lowest in winter.

Scorpion stings are primarily due to accidental contact with scorpion. They use their stings only when roughly handled or trodded on. Scorpion does not always inject venom when it stings since it can control its ejaculation; thus the sting is total, partial or non-existent. Scorpions capable of inflicting fatal stings in humans are all members of families Buthus and Scorpionidae. Reports from Bellary, Rayalaseema, rural Maharashtra, Pondicherry and Chennai have documented such fatalities in children and adults [2].

Species differences, venom dose/weight relationship determine the toxicity and clinical picture. Changes in body temperature may increase the sensitivity of venom and influence the course of toxicity. In India, Israel, Brazil and Mexico cardiac manifestations are common; in Iran tissue necrosis and hemolysis; in South Africa and USA neurological features and in Trinidad acute pancreatitis dominate the picture. Symptoms after scorpion sting progress to a maximal severity in about five hours and subside within a day or two [4].

\section{Aims \& Objectives}

To study the clinical presentation, course, complications and outcome of scorpion sting envenomation. To study the epidemiology and circumstances leading to scorpion sting in the community.

\section{Material \& Methods}

Source of data: All the children admitted for scorpion sting in Mamata General Hospital, Khammam. To verify the epidemiological factors leading to the scorpion sting and the factors predisposing to prevalence of scorpion sting in the community.

\section{Method of collection of data}

Study group: All the children admitted for scorpion sting in Mamata General Hospital, Khammam during the period of 2 years formed the study group.

Inclusion criteria: All cases of definite scorpion sting in children upto 15 years of age in which a scorpion was seen in the vicinity either by the patient or the parents, immediately after the sting. Children with history of bite coupled with classic clinical manifestations of scorpion sting were also included in the study.

Exclusion criteria: Cases of scorpion sting in patients $>15$ yrs of age. Unknown bites and cases where the clinical manifestation was not compatible with scorpion sting envenomation were excluded.

Study design: 50 cases of scorpion sting, admitted to Mamata General Hospital, Khammam from October 2011 to September 2013 were included in the study. On admission, a detailed clinical history, including the time of sting, symptomatology, details of treatment received before admission was taken. Further a description of the scorpion and details about the circumstances leading up to the sting were obtained.

All the patients were subjected to a detailed clinical examination at admission and at frequent intervals thereafter, as was necessary in each case. Hourly monitoring of heart rate, respiratory rate, blood pressure, urine output, cardiovascular and respiratory status was done.

Routine investigations like complete blood counts, peripheral smear, urine routine, bleeding time, clotting time, blood sugar and serum amylase levels, was done in all the cases. Chest radiograph was 
done in cases with evidence of myocarditis or pulmonary edema. Electrocardiography (ECG) and Echocardiography was done in cases with myocarditis and congestive cardiac failure. Computed tomography (CT scan) of the brain was performed in cases with neurological involvement.

Severity of cases was graded as follows: [5]

Grade I : Isolated pain

Grade II : (Systemic manifestations) Hypertension Sweating

Vomiting

Priapism

Shivering

Grade III : (Life - threatening manifestations)

Cardiogenic shock

Pulmonary edema

Altered consciousness
All patients who were symptomatic, received a dose of prazosin (30 microgm $/ \mathrm{kg} / \mathrm{dose}$ ), at admission. Children with peripheral circulatory failure were treated with prazosin, intravenous fluids, and intravenous diazepam $(0.2 \mathrm{mg} / \mathrm{kg})$.

Prazosin was repeated every 4 hours, till peripheries became warm and urine output improved. Myocarditis with congestive cardiac failure was treated with oxygen $(0.5-2 \mathrm{~L} / \mathrm{min})$, maintainence IV fluids, prazosin $30 \mathrm{microgm} / \mathrm{kg} / \mathrm{dose}$ (Nasogastric tube/oral) and Dobutamine infusion (5$15 \mathrm{ig} / \mathrm{kg} / \mathrm{min})$.

Pulmonary edema was treated with oxygen, prazosin, dobutamine infusions, furosemide and by mechanical ventilation, when indicated. All the cases were closely monitored for complications and managed accordingly.

\section{Results}

About 100 cases were included in the study out of which 4-6 years (38\%) age group was the most affected (table1). The youngest case noted was a 2 month old infant and the oldest was a 15 year old child. Scorpion sting were more common in males (64\%). Maximum number of cases were seen in rainy and in the summer season

Table-1: Age wise distribution of cases.

\begin{tabular}{|c|c|c|}
\hline Age & No. of Cases & Percentage \\
\hline$<$ 1 year & 4 & 8 \\
\hline 1 year - 3 years & 4 & 8 \\
\hline 4 years - 6 years & 19 & 16 \\
\hline 7 years- 9 years & 8 & 16 \\
\hline 10 years- 12 years & 8 & 14 \\
\hline 13 years- 15 years & 7 & $\mathbf{1 0 0}$ \\
\hline Total & $\mathbf{5 0}$ & 38 \\
\hline
\end{tabular}

Stings were sustained more in rural areas. Overall, Indoor stings were slightly more common than outdoor stings. Outdoor stings were more common in males (5 6\%), while females were stung mostly indoors ( $72 \%)$.

The housing was Kuchcha in 35 out of the 50 cases $(70 \%)$ of scorpion sting. Based on socio-economic status (kuppuswamy classification) cases were noted among all classes except Class I. Class IV and Class V together accounted for around $72 \%$ of the cases.

The maximum number of cases were stung, when working or playing in the fields.( $48 \%$ ) Among the Indoor stings, stings in cradle or cloth swings (Hammocks) used to put infants to sleep and stings that occurred while children slept on the floor were the most common.

Stings due to Mesobuthus species (Red scorpion) were slightly more common (56\%) than stings due to Palamneus species (Black scorpion). Stings during the day (72\%) were more common than stings during the night. Majority of stings were sustained on the lower extremities (52\%) followed by $24 \%$ in upper extremities, $14 \%$ on trunk and $10 \%$ on face $\&$ scalp 13 children $(26 \%)$ received a dose of prazosin, before admission to the hospital. A similar number received analgesics. Diazepam was given in the periphery to 3 children (6\%). 26 children (52\%) received no treatment and were directly referred to our hospital. 
Pain at the sting site, diaphoresis and restlessness were the most common presenting symptoms. (Table 2)

Table-2: Frequency of Presenting Symptoms.

\begin{tabular}{|c|c|c|}
\hline Symptoms & No. of Cases & Percentage \\
\hline Pain at site of sting & 50 & 100 \\
\hline Salivation & 22 & 44 \\
\hline Diaphoresis & 46 & 42 \\
\hline Vomiting & 20 & 50 \\
\hline Swelling & 25 & 32 \\
\hline Dyspnoea & 16 & 8 \\
\hline Pain Abdomen & 4 & 4 \\
\hline Fever & 2 & 88 \\
\hline Restlessness & 44 & 4 \\
\hline Altered Sensorium & 2 & 2 \\
\hline Convulsion & 1 & \\
\hline
\end{tabular}

The common physical signs noted were, Restlessness, Cold extremities, Tachycardia with Hypotension (68\%) and Tachypnea (24\%). Priapism was noted in $8 \%$ and $10 \%$ had Hypertension. One child had left sided hemiparesis.

Commonest complications were peripheral circulatory failure, pulmonary edema, Myocarditis and Congestive cardiac failure ( $18 \%$ of cases). (table 3$)$

Table- 3: Complications following scorpion bite.

\begin{tabular}{|c|c|c|}
\hline Complications & No. of Cases & Percentage \\
\hline Peripheral Circulatory Failure & 40 & 80 \\
\hline Myocarditis & 9 & 18 \\
\hline Congestive cardiac Failure & 9 & 18 \\
\hline Pulmonary oedema & 12 & 6 \\
\hline Encephalopathy & 3 & 2 \\
\hline Popliteal Artery Thrombosis & 1 & 2 \\
\hline Cerebral Infarct & 1 & 24 \\
\hline
\end{tabular}

Based on severity: Maximum number of cases presented in Grade II (52\%), while $28 \%$ presented in Grade III and $20 \%$ in Grade I

41 cases were admitted with autonomic storm 2 of these children had massive pulmonary edema and succumbed, by 1 hour and 5 hours respectively, in spite of treatment. In the remaining cases, autonomic storm was reversed, during a variable period of 6 hours $(4 \%)$ to 50 hours $(4 \%)$.

ECG changes were noted in $48 \%$ of cases, with sinus tachycardia being the commonest finding (40\%). $4 \%$ had ST elevation / depression and 4\% had $\mathrm{T}$ wave inversion. Chest x-ray revealed cardiomegaly and/or features of pulmonary edema in $24 \%$ of cases. ECHO changes like, left ventricular dilatation of decreased LVEF was noted in $8 \%$ of cases.

Duration of hospital stay: 2 cases succumbed to the envenomation one at 1 hour and the other 5 hours after admission. The duration of hospital stay in other cases varied from 2 day to 14 days, with a maximum of 25 children, requiring a hospital stay of 3 days.

The immediate short-term prognosis was studied in the 50 cases, till they were discharged. A majority of cases recovered without any sequelae. Two cases expired, within hours of admission, due to massive pulmonary edema. One child, who developed popliteal artery thrombosis, 72 hours after the sting, had residual foot drop on the left side and 1 child with right sided cerebral infarct, had left sided hemiparesis, on discharge. 
The incidence of complication was more in younger children. The incidence of complications was slightly more in males than females. Stings due to Mesobuthus species (Red scorpion) seems to carry a increased risk of complication compared to Palamneus species (Black scorpion).

Complications were noted less frequently in children, who received a dose of prazosin early, within $4-8$ hours after sting, compared to those children, who received prazosin late.

\section{Discussion}

Scorpion sting is an acute life threatening, timelimiting medical emergency of villages. Numerous envenomations go unreported and the true incidence is not known [2]. Dominant clinical effects vary from species to species and from one geographical location to another [5]. Case fatality rates vary widely among different regions from $3-22 \%$ and over the years, with improvement in management protocols, there has been a dramatic reduction in mortality [2].

The proportion of cases in the $0-3$ year, $4-6$ years, $7-12$ years and beyond 13 years age groups were $16 \%, 38 \%, 32 \%$ and $14 \%$ respectively. Children aged between 6-12 years are more exploratory and tend to wander outside homes in the darkness and hence are more susceptible to stings [6]. Studies in the past have also shown that most of the admissions for scorpion sting, in pediatric departments are in children between $1-10$ years of age [7].

There was a male preponderance in this study. This has also been noted in the past by various authors [8,9]. This could be because boys, especially toddlers, tend to be more exploratory and wander outside. Further, older boys are frequently employed as agricultural labourers, thus exposing them to field related scorpion stings [10].

Clustering of cases was noted in the summer months $(22 \%)$ and in the rainy months $(30 \%)$. No study has documented the seasonal pattern of scorpion sting, but it is widely observed that cases of scorpion stings increase dramatically in summer and are lowest in winter [2]. This is in keeping with the hibernatory behaviour of scorpions in winter. Scorpions tend to creep out of the burrows in summer, thus increasing the risk of accidental human contact and thus leading to an increased incidence of stings [5].

The incidence of scorpion sting is higher in children living in Kuchcha houses. Kuchcha houses have mud floors and walls and thatched roofs. Scorpions inhabit the crevices and underground burrows in dwellings and these houses provide a safe haven for them. In contrast, Pukka houses with tiled floors and cemented walls and roofs are safer [4]. A higher incidence of sting was noted in lower socio economic groups. The high incidence of stings in this group, is probably due to the type of housing and to their predominantly agricultural presents [5].

A majority of cases (72\%) were from rural areas. Scorpion sting is mainly a rural emergency with habitats of scorpions being primarily, paddy fields, sugarcane, coconut and banana plantations [2].

The proportion of scorpion stings, sustained indoor were slightly more to that sustained outdoors. However, female children and children from urban areas were more likely to be stung indoors, when compared to male children from rural population. Rural male children, are more often involved in agricultural activities and hence are more at risk of accidental contacts with scorpions in the fields. This could explain the high incidence of stings sustained outdoors in them.

Most of the stings sustained outdoors, were in the fields $(48 \%)$, when children accidentally trod over or handled the scorpion and were stung. Bare-foot walking also increased the risk of sustaining a sting. Stings sustained indoor, were mostly when children were sleeping on the floor. Infants were stung, while sleeping in a cradle or a swing made of cloth and hung on the roof (Hammock). Stings also occurred when scorpions were hidden in clothes and in poorly lighted rooms. Outdoor stings are more common than indoor stings in all parts of the world $[5,11]$. However we noted a significant number of indoor stings especially in the urban areas and in females. Further, a number of stings in infants were related to the cradles and hammocks used to put babies to sleep. This should be considered when suggesting appropriate measures for prevention of scorpion stings. 
Stings due to Mesobuthus species (Red scorpion) were slightly more common than those due to Palamneus species (Black scorpion). This preponderance of stings due to Mesobuthus species, in hospital admissions, has been reported in the past $[5,10]$. This could be because of an increased prevalence of scorpions of the Mesobuthus species. Further, scorpions of this species being more venomous,could result in increased rates of hospitalization in children with stings due to this species [6].

Day time stings were more common, in our study. This is in contrast to earlier studies, which showed a preponderance of stings sustained during night time due to nocturnal habits of the scorpion [1,2]. This could be because a significant proportion of stings in our study were sustained outdoors while engaged in agriculture-related activities which is a day-time occurrence.

Although any part of the body can be exposed to sting, in $76 \%$ of cases in our study, the sting was sustained on the extremities. This is comparable to many studies in the past which showed an increased incidence of stings on the peripheries of $60-80 \%$ $[4,11]$. Most of the cases in our study, were stung when accidentally trodding over or handling scorpion in fields or in poorly lighted rooms. Thus, most of the stings were sustained on extremities.

Pain at the site of sting was the commonest complaint noted and was invariably present in all the cases. The pain was usually mild. The high incidence of pain was also noted in previous studies $[5,6]$. Other common symptom noted were profuse sweating, restlessness, vomiting and excessive salivation.

Common physical signs noted were restlessness, cold peripheries, tachycardia and hypotension. $82 \%$ of cases presented with "Autonomic storm", characterized by cold extremities, tachycardia and hypotension. Hypertension was noted in $10 \%$ of cases. Incidence of hypertension in scorpion stings in Indian studies, varies from $12.6 \%$ to $29 \%$ and hypertension is seen usually within 4-8 hours after the sting [5].

Complications were noted in $82 \%$ of the cases, with most of the complications being related to the effects of autonomic storm. Peripheral circulatory failure (PCF) was the commonest complication encountered and is a consequence of fluid loss in the initial cholinergic storm and also secondary to myocarditis [2]. High incidence of Peripheral circulatory failure, ranging from $56-80 \%$ has been noted in various case series in India [5, 12]. Most of the cases of Peripheral circulatory failure responded well to prazosin, fluid resuscitation and inotropic support.

Pulmonary oedema was noted in $24 \%$ of the cases in our study. The reported incidence of pulmonary oedema secondary to scorpion sting in India is around 5\% [5,12]. Although, a high incidence, similar to that seen in our study has been reported before [7].

Popliteal artery thrombosis was an interesting finding noted in a 14 years old male child, stung by a red scorpion. Child was treated with heparin and oral aspirin. The child improved gradually, the lower limb pulses reappeared and pain subsided. On discharge, child had mild residual foot drop of left lower limb. Popliteal artery thrombosis is an extremely rare occurrence following scorpion sting and has not been reported in world literature to the best of our knowledge, though occurrence of deep venous thrombosis has been well reported [5].

Complications were encountered more frequently in younger children. Similar findings were observed by other authors $[6,7]$. The dose of the venom relative to the weight is obviously higher in younger children, thus rendering them susceptible to more severe envenomation and greater risk of complications [2].

The incidence of complications in males was slightly more than females. Bawaskar.H.S et al have reported an increased risk of pulmonary oedema and hypertension in males compared to females [6].

Complications were more common in stings by Mesobuthus sps than Palamneus sps. The clinical effects and toxicity of scorpion venom varies considerably from species to species. Cardiovascular effects are particularly prominent following stings by Mesobuthus tamulus sps [2]. No published data, comparing the rate of complications in stings due to different species is available. Further, the identification of species of scorpion in our study was based on information provided by the bystanders 
and the victim, which may not always be reliable. A more objective study of the species of scorpion would be necessary before drawing any further conclusions.

Stings on the face and scalp had a higher incidence of complications compared to stings elsewhere. There is a paucity of literature relating the rate of complications to the site of sting. In a study published in Saudi Arabia, no significant relationship was found between the site of sting and toxicity [13]. However, closer proximity of sting to head and torso results inquicker venom absorption into the central circulation [3]. This could explain the findings noted in our study.

All the cases admitted, received a dose of prazosin, at admission and every 4 hours thence, till autonomic storm was reversed. Complications were noted less frequently in children who received a dose of prazosin early (within 4-8hrs of sting). This finding is comparable to studies done elsewhere in India, which show that early and effective administration of prazosin significantly reduced the incidence of complications and mortality $[1,14,15,16]$.

Prazosin, an alpha adrenoceptor antagonist is a physiological and pharmacological antidote of scorpion venom [10]. Cardiovascular morbidity and mortality depends on the time interval between sting and administration of prazosin [10]

Two of the fifty study cases expired, giving a case fatality rate of $4 \%$. Both the deaths occurred in younger girls and both had myocarditis, cardiogenic shock, massive pulmonary oedema and encephalopathy. The mortality due to scorpion sting has dramatically declined over the years from upto $68 \%$ to less than $1 \%$. $[2,4]$.

Deaths due to scorpion sting occur mainly due to massive pulmonary oedema, CCF with cardiogenic shock or recurrent seizures $[5,12,13]$. Improved management practices and early administration of prazosin are the important factors responsible for the decline [17].

The relatively higher incidence of mortality noted in our study, is due to delayed referral and failure to seek medical care early. The delay in administration of prazosin (more than 24hrs) was significant and could have contributed to development of severe complications. Our experience highlights the importance of a prompt referral to a tertiary centre and early administration (within 4 hrs of sting) of prazosin in the periphery to avert such tragedies in the future,

\section{Conclusion}

Scorpions have been known to man since time immemorial and have a significant presence in mythology and human history. Scorpion stings, once considered just a painful nuisance have now got the attention and care that they deserve. The relentless invasion of man into the habitats of scorpions has brought us in direct conflict with these Arachnids. Scorpion stings are an "occupational hazard" for children from rural areas, employed in agricultural pursuits.

They constitute a significant public health problem in many underdeveloped, tropical and subtropical countries, including India. In India, cardiovascular complications are most common and life threatening. However, anticipation and close monitoring for other uncommon complications is critical for effective management. Prazosin has revolutionized the management of scorpion sting envenomation.

Administration of prazosin, as early as possible, is probably the single most effective intervention for preventing complications following scorpion stings. Early and effective prazosin therapy, good supportive care, close monitoring and management of complications can limit the resulting morbidity and mortality significantly.

The role of scorpion antivenom still remains controversial. Public awareness regarding measures for prevention of sting and physician readiness to combat this common emergency can go a long way in preventing the devastating effects of this condition.

Funding: Nil, Conflict of interest: None initiated, Perission from IRB: Yes

\section{References}

1. Clark RF. Scorpion Envenomation. In: Clinical Toxicology. Ford MD, Delaney KA, Ling LJ, Erickson J, edt., 1st Edn,. W.B. Saunders company; Pennsylvania:2004,p.290-93. 
2. Mahadevan S. Scorpion sting. Indian Pediatr. 2000 May;37(5):504-14.

3. Himmatrao SalubaBawaskar, Pramodini Himmatrao Bawaskar. Scorpion Sting: Update. JAPI January 2012;60(1):46-55.

4. Mahaba HMA. Scorpion Sting Syndrome : Epidemiology, clinical presentation and management of 2240 cases. Eastern Mediterranean Health J 1997;3(1):82-89.

5. Mahadevan S. Scorpion Sting. In: Principles of Pediatric and Neonatal Emergencies. Sachdev HPS, Choudhury P, Bagga A, Chugh K, Ramji S, Puri RKedt.,2nd edn., Jaypee Brothers; New Delhi: 2003, p.409-15.

6. Bawaskar HS, Bawaskar PH. Indian red scorpion envenoming. Indian J Pediatr. 1998 May-Jun;65 (3): 383-91.

7. Biswal N, Bashir RA, Murmu UC, Mathai B, Balachander J, Srinivasan S. Outcome of scorpion sting envenomation after a protocol guided therapy. Indian J Pediatr. 2006 Jul;73(7):577-82.

8. Cheng D, Dattaro JA, Yakobi R. Scorpion sting.Cited on 2005 June 23; 24 screens: Available from: www.emedicine.com/med/topic 2081.html

9. Santhanakrishnan BR, Gajalakshmi BS. Pathogenesis of cardiovascular complications in children following scorpion envenoming. Ann Trop Pediatr1986;6(2):117-121.
10. Kulkarni ML, Anees S. Snake venom poisoning: experience with 633 cases. Indian Pediatr. 1994 Oct; 31 (10):1239-43.

11. Adiguzel $\mathrm{S}$, Ozkan $\mathrm{O}$, Inceoglu $\mathrm{B}$. Epidemiological and clinical characteristics of scorpionism in children in Sanliurfa, Turkey. Toxicon.2007 May;49(6):875-80. Epub 2007 Jan 10.

12. Santhanakrishnan BR. Scorpion sting. Indian Pediatr. 2000 Oct;37(10):1154-7.

13. Bogomolski-Yahalom V, Amitai Y, Stalnikowicz R. Paresthesia in envenomation by the scorpion Leiurus quinquestriatus. J Toxicol Clin Toxicol. 1995;33 (1):79-82.

14. Bawaskar HS, Bawaskar PH. Clinical profile of severe scorpion envenomation in children at rural setting. Indian Paediatr 2003; 40(11):1072-1076.

15. Bawaskar HS. Management of severe scorpion sting at Rural settings: What is the role of scorpion antivenom? J. Venom Anim Toxins incl Trop Dis 2005; 11(1):3-7.

16. Bawaskar HS, Bawaskar PH. Prazosin in the management of cardiovascular manifestations of scorpion sting. Lancet 1986;1(8479):510-11.

17. SinghalA., MannanR., RampalU. Epidemiology, clinical presentation and final outcome of patients with scorpion bite. Journal of clinical and diagnostic research 2009; 3(3):1523-1528.

\section{How to cite this article?}

Reddy R.M, G. Somaiah. Clinical and epidemiological study of scorpion sting envenomation at a teaching hospital in rural Telangana. J PediatrRes.2017;4(07):461-468.doi:10.17511/ijpr.2017.i07.05. 